\title{
Sistema difuso para la detección y diagnóstico de falla en la generación a vapor
}

\author{
Fuzzy system for the detection and diagnosis of failure to steam generation
}

\author{
${ }^{1}$ Marlon Hernandez Cely, ${ }^{2}$ Alexander Sandoval Estupiñan, ${ }^{3}$ Fabián Valencia Rivera \\ ${ }^{1,2,3}$ Grupo SIDDFA, Universidad Francisco de Paula Santander, Cúcuta ,Colombia \\ marlon25_3@hotmail.com \\ alexandersandoval_1987@hotmail.com \\ favalencia-89ehotmail.com
}

\begin{abstract}
Resumen - En la industria existen procesos que necesitan de generadores a vapor para su producción y cuando estos fallan generan pérdidas, por esta razón es necesario desarrollar un sistema de diagnostico de fallas SDDF fundamentado en lógica borrosa para mejorar la eficiencia del proceso y así evitar paros no programados en la producción. Mediante el modelado difuso, se analiza el comportamiento de falla, ante diferentes patrones o esquemas de funcionamiento aplicando metodologías no basadas en el conocimiento del modelo, integrado al sistema de seguridad en calderas, ejecutando de forma directa y eficaz la predicción de las fallas presentes en el proceso.
\end{abstract}

Palabras clave- Control difuso, SDDF, Simulaciones, Sistemas de generación a vapor.

Abstract-In industry there are processes that require steam generators for their production and generate losses when they fail, therefore it is necessary to develop a SDDF based on fuzzy logic to improve process efficiency and avoid unscheduled shutdowns in production. Using the fuzzy modeling, we analyze the behavior of the fault, with different patterns or patterns of operation using methods not based on knowledge of the model, integrated security system in boilers, running in a direct and effective prediction of faults in the process.

Key Word - Fuzzy control, SDDF, Simulations, steam generation systems.

\section{INTRODUCCIÓN}

Los Sistemas de Detección y Diagnóstico de Fallas (SDDF) actualmente son una necesidad en la industria. Cada día los procesos se hacen más complejos y el riesgo de que se produzca una catástrofe ocasionada por fallas es cada vez mayor.
En este caso el mal funcionamiento de ciertos sistemas como es el generador de vapor comienza a tener un gran impacto económico incluso para pequeñas desviaciones de su comportamiento con respecto al esperado por diseño [1]. De presentarse una falla y no ser atendida a tiempo en principio ocasiona degradación del equipo, fallas en el servicio, pérdidas económicas pero principalmente peligro para la integridad del operador. Con el fin de mantener altos niveles de confiabilidad y seguridad.

Estas situaciones de complejidad han llevado a la búsqueda de nuevas estrategias tratando de dar una solución satisfactoria. Mediante el empleo de la teoría de la LOGICA DIFUSA es describir y formalizar la realidad, empleando modelos flexibles que interpreten las leyes que rigen el comportamiento humano y las relaciones entre los hombres.

En la caldera de Termotasajero Colgener durante los últimos años las fallas más comunes han sido roturas de tubo generados por la no existencia de un control eficiente en algunos factores importantes como la presión, el nivel y la temperatura.

- Planteando posibles soluciones para evitar estas fallas, se utilizara la lógica borrosa como sistema control de las alzas o bajas de temperatura, presión o nivel.

\section{CONTENIDO}

\section{A. Sistemas de generación a vapor}

El sistema de generación a vapora utilizado para nuestra investigación son calderas Acuatubulares que utiliza como combustible principal carbón en industrias termoeléctrica [2], [3]. 


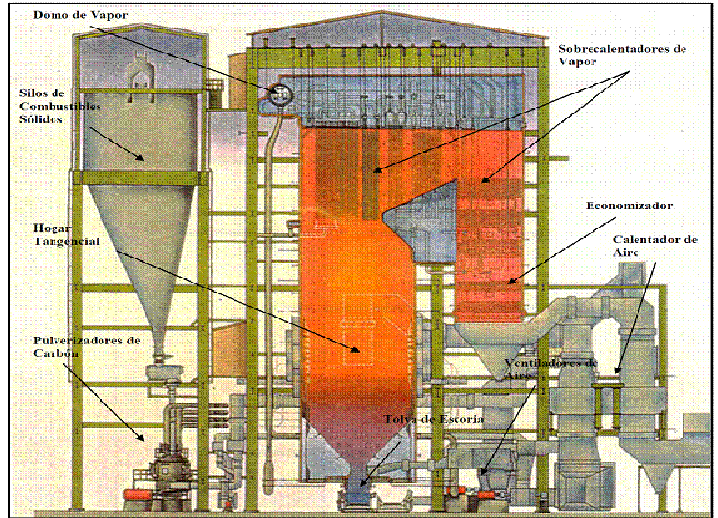

Figura 1. Sistema de calefacción por vapor.

Un sistema de calefacción a vapor tiene el siguiente funcionamiento, Ver Figura 2.

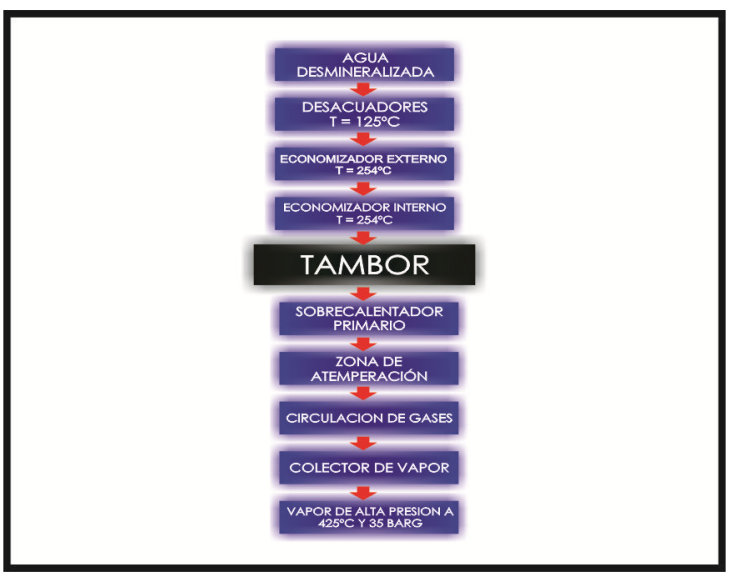

Figura 2. Diagrama en bloques del proceso de generación a vapor.

B. SDDF mediante sistemas automático difuso

Fundamento: Aproximaciones basadas en reglas que han sido propuestas como un método capaz de realizar detección y diagnóstico flexible. Las reglas son descritas como la relación entre las causas y los síntomas de las fallas. [4], [5].

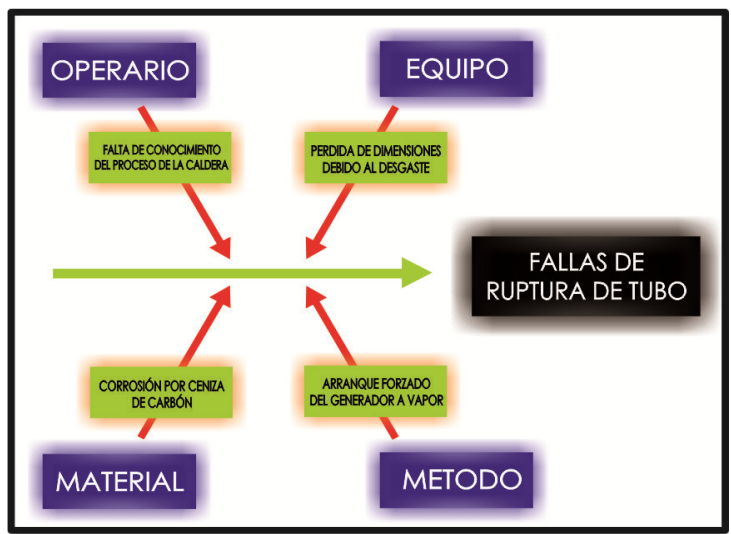

Figura 3. Modelo de falla de rotura en tubo.
El conocimiento elegido que permite la interpretación y el diagnóstico, está organizado en la base de conocimiento como un conjunto de estados condicionales difusos, que relaciona los resultados de la prueba con las conclusiones acerca de las condiciones del proceso o de las posibles fallas [6].

\section{Identificación de fallas}

Cuando se configura el sistema de inferencia difusa, se efectúa la evaluación de las entradas (valores provenientes de los indicadores en planta) mediante el proceso de fusificación (obtención de los grados de pertenencia de los valores a las funciones de pertenencia), luego la implicación empleando la base de reglas y finalmente la Desfusificación (obtención de los valores numéricos a partir de los conjuntos difusos). El resultado de la Desfusificación es un conjunto de indicadores de distintas áreas del proceso, estos valores son interpretados por el sistema [7].

La interpretación de salidas emplea una base de vínculos, cada salida (indicador) tiene un conjunto de vínculos asociados, la estructura de un vínculo se muestra en la figura 4. [7], [8], [9].

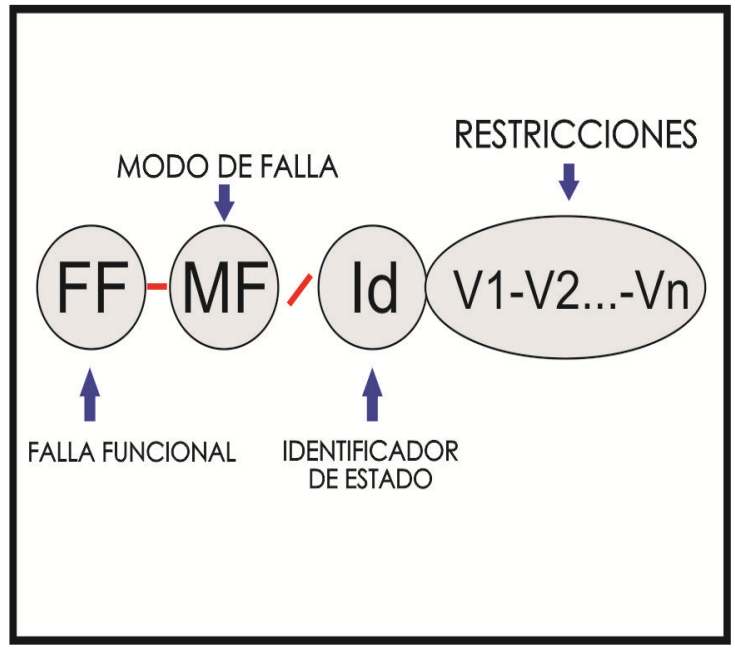

Figura 4. Estructura de un vínculo. 


\section{Simulaciones}

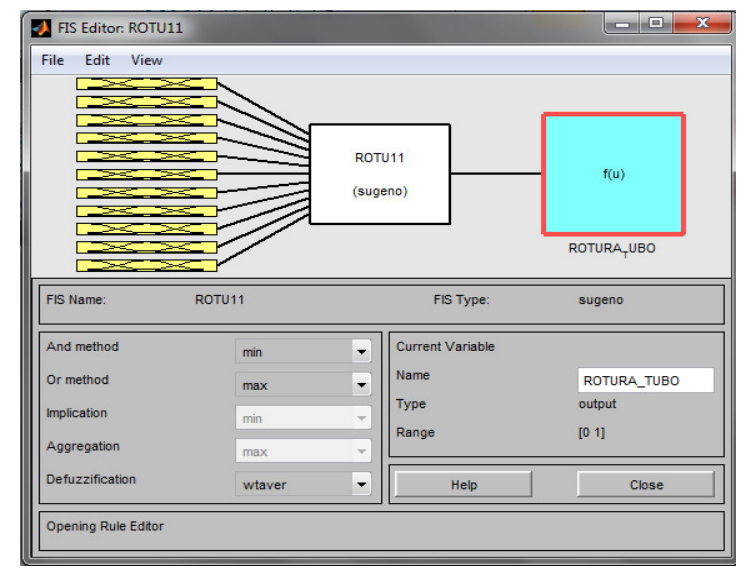

FIGURA 5. Programa de control lógico difuso para diagnóstico de rotura de tubo.

\section{Entradas en $S D D F$}

Las entradas son las variables empleadas para la supervisión de la presión en el domo y el sobrecalentador y la temperatura en el tubo. Para configurar las entradas el sistema toma como información los valores de operación normal de las variables de planta consideradas como entradas a fusificar, así como las condiciones de falla, con esta información se definen los rasgos de las funciones de pertenencia y el universo de discurso las entradas son [7], [8], [9], [10]:

- T1: PSH 51401. Alarma alta presión hogar con un parámetro de $106 \mathrm{~mm} \mathrm{H}_{28}$.

- T2: LT-81202 Transmisor de nivel, alarma por bajo nivel del domo con un valor de $-100 \mathrm{~mm}_{\mathbf{H}_{2 \circ}}$.

- T3: TC 71202 Controlador de temperatura, alta temperatura salida Pulverizador $930^{\circ} \mathrm{C}$.

- T4: FT-.61203 Transmisor de flujo, flujo de agua de alimentación, con una medida de de 0 a $600 \mathrm{~mm} \mathrm{H}_{20}$.

- T5: PT 51207 Transmisor de presión aire primario con un parámetro de $630 \mathrm{~mm} \mathrm{H}_{2 a}$

- T6: PT 51239 Transmisor de presión, alta presión vapor principal $128 \mathrm{Kg} / \mathrm{cm}^{2}$.

- T7: TF-61209. Transmisor de flujo, decremento en la producción del vapor principal.

- T8: PSH 51402. Disparo de la caldera por alta presión en el hogar con un valor de $200 \mathrm{~mm} \mathrm{H}_{30}$.

- T9: PX 208. Transmisor de Presión de vapor salida a caldera $0-128 \mathrm{Kg} / \mathrm{cm}^{2}$.

- T10: LT- 81201 Transmisor de nivel, la caldera queda sin servicio por muy bajo nivel con valor de $-253 \mathrm{~mm}$ $\mathrm{H}_{20}$.
- T11: TC 71201Control de temperatura. Alta temperatura vapor sobrecalentado.

Valores Lingüísticos para las Entradas del SDDF en el Domo[11], [12]:

- MB: Muy Bajo

- B: Bajo

- BA: Bajo Alto

- BM: Medio Bajo

- ME: Medio Estable

- M: Medio

- EN: Estado Normal

- MA: Medio Alto

- AB: Alto Bajo

- ABM: Alto Bajo Medio

- AM: Alto medio

- AMA: Alto Medio Alto

- AS: Alto Superior

- AO: Alto Optimo

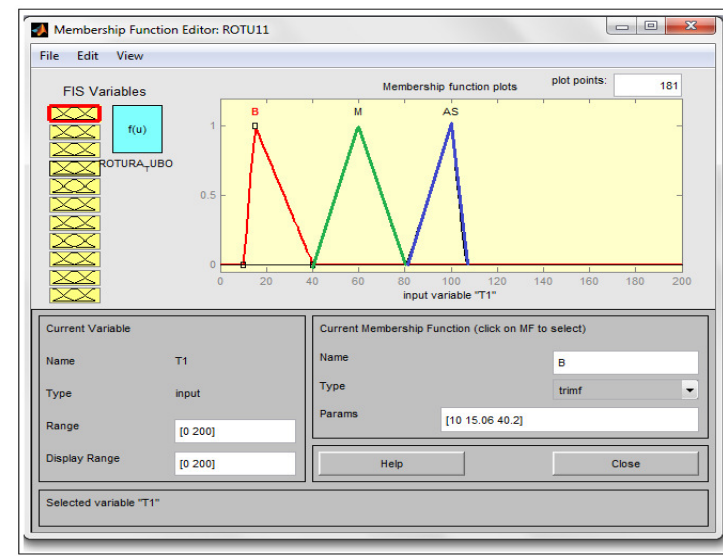

Figura 6. Funciones de membrecía.

\section{Salidas en SDDF:}

Para la definición de las variables lingüísticas de salida resultante del proceso de inferencia (evaluación de proporciones), se definieron variables que cumplieran con la función de ser indicadores de estados anormales para la ruptura de tubo. La caracterización de las variables linguísticas se hace definiendo estados anormales los cuales se mencionan a continuación. Las salidas para el SDDF son:

- Ruptura_tubo: Ruptura del tubo

Valores Lingüísticos para las Salidas del SDDF:

- P2: Flujo de carbón (Pulverizadores)

- P3: Flujo Vapor Sobrecalentado.

- P4: Presión Salida Recalentador.

- P5: Temperatura Salida Recalentador. 
- P7: Presión de salida vapor sobrecalentado

- P8: Temperatura de salida de vapor sobrecalentado

- P9: Alta presión en el hogar.

- P10: baja presión del domo

- P11: Bajo nivel domo.

- P12: Rotura de tubo generador de vapor.

- P17: Temperatura Entrada Recalentador.

- P18: Presión Entrada Sobrecalentador.

- P19: Flujo de agua.

- P20: Flujo de aire

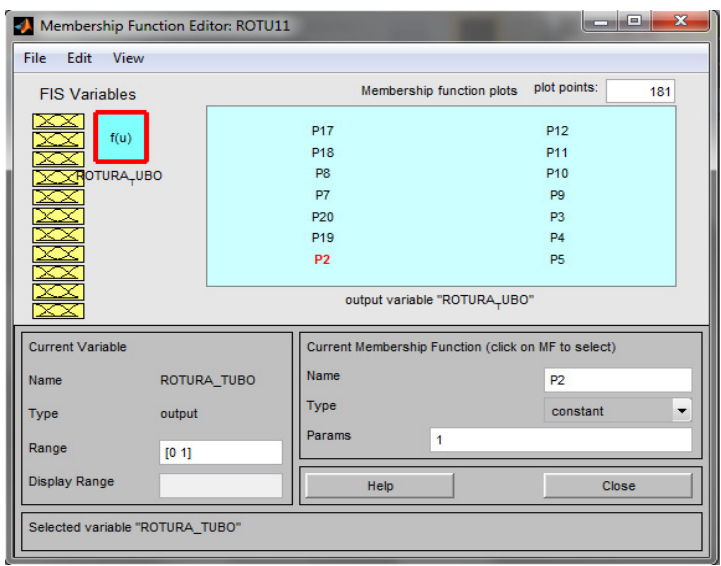

Figura 7. Salidas del SDDF para ruptura del tubo.

Generación de las leyes

En la gráfica tenemos el Rule Editor de MATLAB en el cual se implementaron las 402 reglas para el sistema de detección y diagnóstico de fallas indicando el nivel de ruptura del tubo presente en el domo, dando como respuesta en escala de 0 a 5 el estado de la ruptura y/o nivel de crisis presente en el tubo

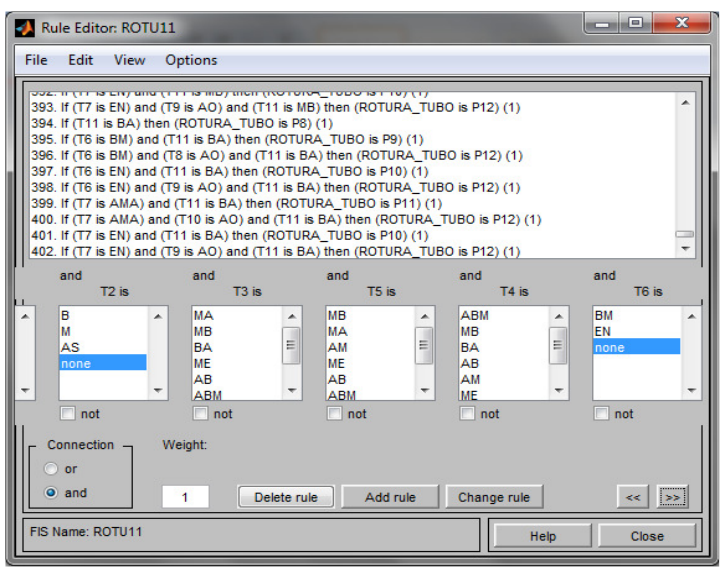

Figura 8. Rule editor: ROTU 11.

Desfusificación del Sistema

Desfusificación del SDDF.Cuando intentamos obtener una solución a un problema de decisión, lo que queremos obtener como salida es un número y no un conjunto difuso [13].

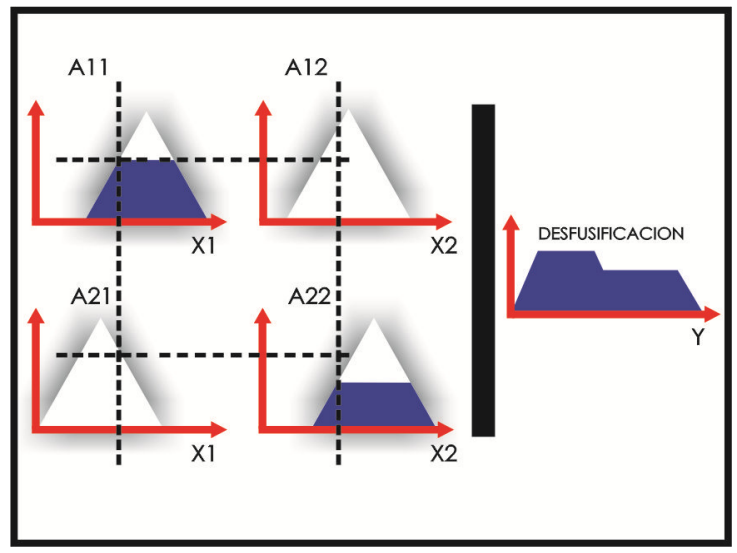

Figura 9. Sistema Difuso Procesamiento Detallado.

Por tanto, Dadas las salidas de los consecuentes individuales $y_{i}$, la salida total $y$ del modelo difuso Takagi-Sugeno (desfusificación o concreción) es calculada usando la ecuación 1.

$$
y=\frac{\mathbb{V}_{i=1} w_{i}(x) y_{i}}{\sum_{i=1} w_{i}(x)}
$$

Ecuación (1)

Una vez programados los sistemas de detección y diagnóstico de falla procederemos a validarlo, la validación se realizara mediante simulaciones.

La simulación de los sistemas de detección y diagnóstico de fallas desarrollados anteriormente se llevó a cabo utilizando las herramientas proporcionadas por el software Simulink de MATLAB y su paquete Toolbox de lógica difusa (Fuzzy Logic Toolbox).

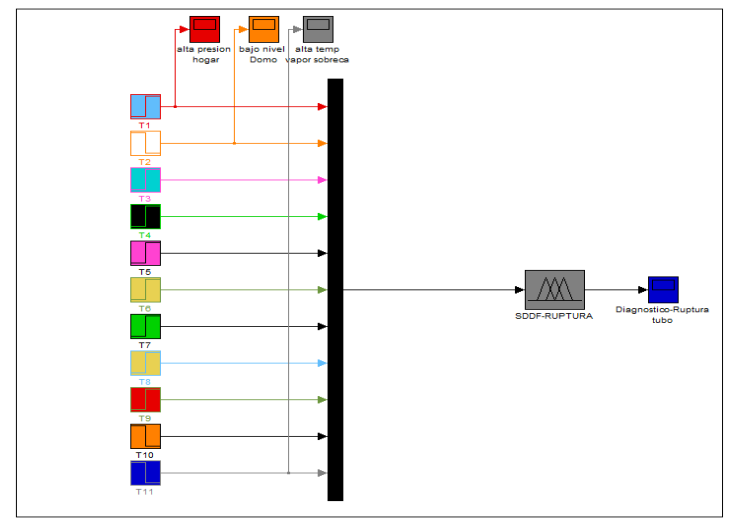

Figura 10. Implementación del diagrama de bloques en simulink.

Para la validacion se simulo el SDDF tomando múltiples valores aleatorios reales para cada una de las entradas y obtener respuestas precisas de la falla. 
Los datos de la entradas son los siguientes valores ver en las graficas

- $\mathrm{T} 1=100$

- $\mathrm{T} 2=90$

- $\mathrm{T} 3=1200$

- $\mathrm{T} 4=650$

- $\mathrm{T} 5=700$

- $\mathrm{T} 6=128$

- $\mathrm{T} 7=650$

- $\mathrm{T} 8=200$

- $\mathrm{T} 9=140$

- $\mathrm{T} 10=-250$

- $\mathrm{T} 11=541$

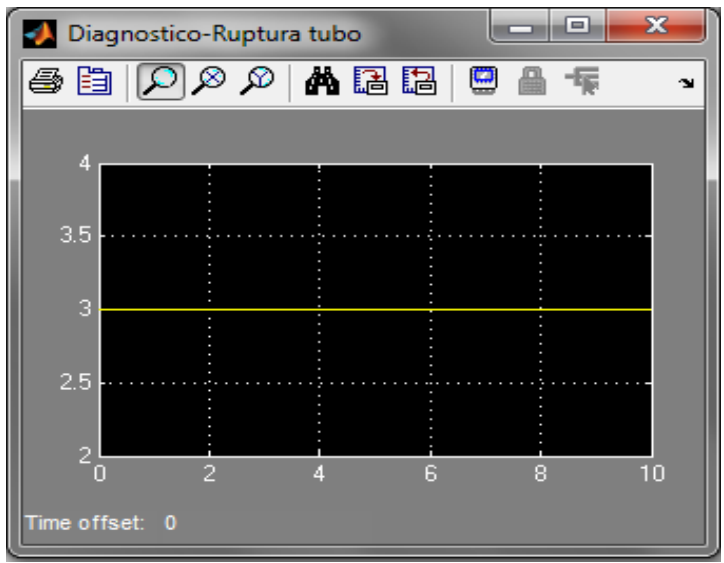

Figura 11. Salida estado SDDF ruptura de tubo.

Una vez simulados tenemos presente en la salida bajo un margen de calificación del SDDF para determinar estado de ruptura del tubo de 0 a 5 :

- 0: apagado

- 1 :encendido

- 2-3:estado normal del tubo

- $\quad$ 4:Presente calentamiento índice de posible ruptura

- 5:falla presente en el tubo

\section{CONCLUSIONES}

La metodología aquí propuesta fue simulada, ejecutada y analizada dando como respuestas satisfactorias. Igualmente esta puede ser expandida para cualquier sistema debido a que es una metodología ajustable que tiene en cuenta información como: La datos históricos, Información de expertos recolectada a través de entrevistas informales, tormenta de ideas y video conferencias, siendo esta una muy buena fuente de información para la aplicación de la metodología permitiendo la toma de decisiones acertadas aumentando las ganancias por selección certera de los estudios de confiabilidad.

En nuestra investigación el SDDF desarrollado en Lógica Borrosa, permite una rápida detección de la falla considerando 
nuestro diseño y los posibles tipos de averías que se pueden presentar en el proceso. Al continuar este trabajo, quedaría hacer un análisis comparativo con otro tipo de metodología no basada en el modelo del conocimiento como: Redes neuronales, Inteligencia artificial y redes de Petri.

Con el modelo cualitativo del proceso de nivel en el domo de la caldera, a través del método basado en Lógica Borrosa. Se pudo modelar y simular (Matlab, simulink, toolbox de la lógica difusa) el comportamiento del SDDF y con ello validar y comprobar la hipótesis plantada en el presente proyecto.

La manera mediante la cual se validaron y socializaron los resultados obtenidos fue a través de la presentación ante ingenieros en el área de la mecánica y electrónica pertenecientes al grupo de trabajo de Termotasajero Colgener, facilitando datos para citar como ejemplo a esta planta termoeléctrica, grupo de estudiantes de la Universidad Francisco de Paula Santander. Los cuales quedaron satisfechos y en total acuerdo con los resultados expuestos. Además la falla se simulo con datos de operación reales del proceso otorgado por el grupo de trabajo de la Termotasajero Colgener.

\section{REFERENCIAS}

\section{$\underline{\text { Referencias de libros: }}$}

[1] Remiro Jesús A. y Lozano Miguel A., Control del Rendimiento y Diagnóstico Termo económico de Centrales Termoeléctricas Información Tecnológica Vol 18(1), GITSE, Dpto. de Ingeniería Mecánica, Zaragoza-España (2007).

[2] KOHAN, Anthony. "Manual de Calderas". Madrid. McGraw-Hill, 2000.

[3] Walter Gastelbondo Barragán, Estudio Para el Incremento del Nivel Integrado De Seguridad (S.I.L.), de los Sistemas Instrumentados de Segurida S.I.S.) Industrial, Mediante Esquemas y Algoritmos Tolerantes a Fallas Utilizando Sistemas de Detección y Diagnóstico de Fallas, Universidad de Pamplona 2007, pag. 17.

\section{Reportes Técnicos:}

[4] Aranguren Sandra, "Estudió y Diseño de las Normas, Ingeniería, Metodologías y Tecnologías y Diagnostico de Fallas (SDDF), para los Sistemas de Automatización Industrial”.

[5] Instituto Colombiano de energía Eléctrica, ICEL Central Termoeléctrica de Tasajero. "Manual de entrenamiento de la Caldera Termotasajero I y sus equipos Auxiliares" Termotasajero 2000.
[6] Tarantino Alvarado Rocco Aranguren Sandra. "Introducción a la detección y diagnóstico de fallas en la industria".2004

Referencias de libro tesis de maestría:

[7] Marlon Mauricio Hernández Cely, Estudio para detección y diagnóstico de fallas en el proceso del generador de vapor de la central termoeléctrica TERMOTASAJERO colgener, universidad de pamplona, 2010

Referencias de libros:

[8] [Kevin M. Passino, Stephen Yurkovich, "Fuzzy Control", Addison-Wesley 1998.

[9] F. Martin McNeill, Ellen Thro, "FUZZY LOGIC A PRACTICAL APPROACH", AP PROFESSIONAL, Boston, 1994.

[10] Castro Mora Javier. "Capacitación para mejorar la competitividad y el desarrollo tecnológico en las plantas térmicas de generación de energía”. Caldera II Marzo 1998.

Reportes Técnicos:

[11] Mina J, Verde C, "Detección de Fallas usando Análisis de Componentes Principales", Instituto de Ingeniera, UNAM CONGRESO ANUAL DE LA AMCA 2004, México.

[12] "Mantenimiento Predictivo-proactivo atreves del análisis del Aceite", Escuela técnica Superior de Ingenieros Industriales, X Semana de Ingeniería, las palmas gran canaria 2002.

$\underline{\text { Referencias de libro tesis de maestría: }}$

[13] Tesis del ing. José Luis López, pradoestudio de metodologías y tecnologías para la aplicación de sistemas de detección y diagnóstico de fallas en los instrumentos del generador de vapor de una central termoeléctrica, universidad de Pamplona, 2010

\section{$\underline{\text { Referencias consultadas de web: }}$}

[14] www.scielo.org.ve/scielo.php?Pid=so254770200500010000 6\&scrip=sci_arttextContiene: librería electrónica científica en línea [Fecha de consulta: 17/11/11].

http://www.edutecne.utn.edu.ar/fuzzy_control/UTNFCONTR.pdf Contiene: sistemas basados en reglas (control fuzzy) 2008 [Fecha de consulta: 28/11/11]. 